\section{Four Dimensions of Rules and Their Correspondence to Rule-Governed Behavior: A Taxonomy}

\author{
Martha Peláez \\ Florida International University \\ and \\ Rafael Moreno \\ University of Seville
}

The taxonomy presented takes into account dimensions of an entire contingency arrangement specified in the rule and how these dimensions relate to the listener's behavior. The classification is made according to rule: (a) explicitness, (b) accuracy, (c) complexity, and (d) source. It is argued that the probability that the listener will behave according to a rule depends on the type of rule provided, the context in which the rule is provided, and listener's history with that or other similar rules. Even though manipulations of other types of rules have been conducted in studies of stimulus equivalence, relational frames, and derived stimulus relations, a systematic study of the differential effects of the proposed 16 types of rules on the listener's behavior is recommended.

Behavior analysts have distinguished rule-gov erned behavior from direct contingency-shaped behavior on the bases of different sets of controlling contingencies (e.g., Galizio, 1979; Reese, 1989; Verplanck, 1992; Zettle \& Hayes, 1982). However, the contingency-specifying verbal stimuli (i.e., the rules), whose function is to control behavior and that have function-altering effects (Schlinger \& Blakely, 1987) have not been systematically analyzed and classified in terms of both form and function. The classification of rules offered here may contribute to an advancement in the structural and functional analysis of rule-governed behavior.

\section{On the Meanings of Rule-Governed Behavior}

Rule-governed behavior has been distinguished theosetically and experimentally from behavior that is shaped and maintained by its direct consequences (e.g., Catania, 1985; Catania, Shimoff, \& Matthews, 1989; Cerutti, 1989; Hineline \& Wanchinsen, 1989; Vaughan, 1989; Zettle \& Hayes,
1982). Skinner $(1953,1957,1966,1969)$ distinguished between behavior shaped by direct consequences, naming it contingency-shaped behavior, and behavior controlled by verbal antecedents, naming it rule-governed behavior. In his account, contingency-shaped behavior is maintained by direct consequences and comes under the control of discriminative stimuli. In contrast, rule-governed behavior, is controlled by verbal behavior, and only indirectly maintained by its consequences. In this sense, Skinner (1966) identified rules as contingency-specifying verbal stimuli--as stimuli that specify, either directly or indirectly, consequences for the behavior.

\section{Rules and Rule-Governed Behavior As Useful Concepts}

A concept of rule-governed behavior can be useful if it accommodates the description of complex behavior that is under the control of contingencies and can be modified by antecedent verbal stimuli (i.e., rules). The primary function of a rule, then, is to influence or guide the behavior of the listener--controlling the listeners' behavior in ways specified by the verbal behavior of the speaker. Such control can include producing novel ways of behaving.

Although the control of rules in governing behavior has been demonstrated, the distinction between contingency-shaped behavior and rule-governed behavior, at times, is unclear. Theoretical inconsistencies in the distinction between notions of contingency-shaped and rule-governed behavior have been discussed (e.g., Cerutti, 1989; Peláez-No gueras \& Gewirtz, 1995; Ribes, 1992).

We should emphasize that although both are established by consequences, the controlling variables and functional properties of contingency-sha ped and rule-governed behavior differ. The particular functions of verbal stimuli, as controlling rules, are to specify (either explicitly or implicitly) the entire contingency array among antecedent stimulus, response, and consequence, in a given context. A rule must be understood in terms of the descriptions it makes of contingent relations among the three-term contingency (four or five term) in context. Such relations might or might not be present in the very situation where the rule is given, which imply more complexity of the entire contingencies embedded in the rule. The transmission of these "non-present complex contingent relations" can be achieved only through language. Clearly, the ultimate controlling charac- 
ter of a rule is based on ready-made discriminative attributes that, by virtue of the listener's verbal history, do not require new conditioning in every new situation in which the rule is provided. Moreover, individuals can behave from the outset in accordance with rules that they have never before encountered.

Zettle and Hayes (1982) stress that rule-govemed behavior involves two sets of contingencies: those related directly to the behavior of interest, and those related to the verbal antecedents of such behavior (i.e., tracking and pliance). From our perspective, the emphasis is in the analysis of the two sets of the entire contingencies involved in terms of both form and function. The two sets of contingencies we are interested in analyzing are those specified in the rule (SD--R-SR) and those in which the listener's behavior is embedded (Sd-R$S R$ ) -the latter contingencies resulting from directacting. Rules and rule-related behaviors can be meaningfully understood only when analyzed as an interdependent unit.

\section{Analyzing the Interdependent Unit}

There exists a co-dependent relation between the rule and the behavior of the listener. A rule's function can only be identified in terms of its relation to rule-governed behavior, and rule-governed behavior makes sense only in reference to a rule, or set of specified contingencies. A rule's form or structure, however, can be identified a priori, be fore identifying the behavior of the listener.

We should stress, though, that the verbal character of rules must not be understood in a strictly morphological sense (i.e., in terms of rule form or structure), given that verbal stimuli of different forms can have an identical functions (e.g., red traffic light, the word "STOP"). In studying rules we must consider both their structure and function. Functionally speaking, then, each contingency set involves a mutually dependent relationship between environmental stimuli and the individual's responses. Each set is influenced by its current and historical context (Kantor, 1924/1926; Morris, 1988; Peláez-Nogueras, 1994). In sum, the probability that the listener will behave according to a rule depends on (a) the contingencies specified in the speaker's rule, (b) the context within which the rule is provided, and (c) the listener's history with that or other similar rules.

In our present analysis, we focus on rules and on the set of contingencies that they specify for the listener (Skinner, 1989). The specifications of con- tingencies embedded in rules result from the speakers' verbal behavior. We should clarify that, we examine rules in terms of the contingencies they specify, although we do not analyze the behavior of the speaker per se (the behavior of the rule-giver), nor the history of contingencies and variables maintaining the speaker's rule-giving behavior. Rule-following behavior is controlled by the results of rule-giving behavior and it is in this sense that we are only indirectly interested in the behavior of the speaker.

Our main purpose in the remainder of this paper is to analyze: (a) the form and function of the contingency specifying verbal stimuli that can control listener's behavior, and, (b) the related listeners' rule-following behavior.

\section{Dimensions of Rules and Their Related Behavior}

A functional identification or classification among different types of rules and their correspondence to rule-governed behavior has been limited. The classification is made according to four dimensions: (a) explicitness, (t) accuracy, (c) complexity, and (d) source.

\section{Explicitness of the Rule}

Explicit versus implicit rules. Rules can be distinguished based on the completeness or specificity of the contingencies expressed by the speaker. The explicit rule clearly identifies the components of the entire contingency and its context. Such explicit rules incorporate all the contingency components, comprehensively specifying readily identifiable contexts. In contrast, in the implicit rule, the contingencies may not gain verbal expression, either because some of the components are unnamed or because they are expressed in a way not identifiable in time and space. An example of an implicit rule omitting components of the entire contingency would be: "Keep your eyes on the road while you are driving," in which the consequence is not specified. In studies of matching-tosample and stimulus equivalence, subjects often follow rules where the antecedent stimulus-response relations are omitted in the instructions provided by the experimenter. Some maxims and proverbs represent examples of implicit rules that name components without assigning them concrete identification. For instance, "A peaceable person is a long-lived one." 
Explicitly versus implicitly rule-govemed behavior. For the most part, correspondence between rule-governed behavior of the listener and the rule provided by the speaker will be determined by the explicitness of the contingencies contained in the rule. The more explicit the elements of the contingency expressed in the rule, the more direct the influence upon the listener's behavior (Martínez, González, Ortíz, \& Carrilio, in press; Martínez, Moreno, Ortíz, \& Carrillo, in press; Trigo, 1998). Nevertheless, the subject can learn to respond to classes of rules characterized by incomplete contingency specification, such as when the subject complies with the rule "Don't do it!," even though entire elements of the contingency and its context lack description. This is possible due to the listener's learning history in a given context.

\section{Accuracy of the Rule}

Accurate versus inaccurate rules. An accurate rule specifies contingencies that, when followed, match certain event-consequence relationship in the environment. Here, accurate rules specify contingencies that may occur. An inaccurate rule describes contingencies that do not correspond to those encountered in the environment. An example of the former is "If you keep looking away while you are speeding, you may have an accident." A rule of the inaccurate case is "If you keep speeding you are going to be rewarded by the police." When the programmed contingency corresponds to the feedback provided by the experimenter, we speak of accurate rules. Conversely, when programmed contingencies do not correspond to or contradict the experimenters' feedback or rules, then we speak of inaccurate rules.

Adjusted versus non-adjusted rule-govemed behavior. The listener's behavior may adjust to the speaker's rules when the contingencies specified in the rules are accurate or correspond to the programmed (or direct) contingencies (Degrandpre \& Buskist, 1991). Rule-govemed be havior is sensitive (or adjusted) to the prescribed contingencies only to the extent that these prescriptions are consistent (or correspond) with the programmed contingencies. Following inaccurate rules may desensitize the listener to the effects of programmed contingencies (Buskist \& Miller, 1986; Catania, Matthews \& Shimoff, 1982; Martinez \& Ribes, 1996; Michel \& Bernstein, 1991) Hence, in addition to the degree of accuracy in a given contingency-rule prescription, the listener's history and current context significantly affect the extent to which rules will govern behavior.

\section{Complexity of the Rule}

Lower versus higher nule complexity. The contingencies specified in a rule always include at least one relation among behavior, its antecedent stimuli, and its consequences. In our analysis, rule complexity refers to the number of dimensions of the antecedent stimuli and their relations (Peláez, Moreno, Martinez, Trigo, \& Qiang, in review). Dimensions are characteristics or attributes of stimuli employed, for instance, in matching procedures. Colors, shapes, sizes, and positions represent dimensions of stimuli and can be related to one another. The taxonomy of rule complexity offered here is organized hierarchically and is inclusionary, meaning that each lower level of complexity forms part of the next higher level.

A rule's lowest level of complexity specifies at least one dimension of a sample stimulus. For example, the instruction: "Name the colors of the figures appearing on the screen" specifies only one dimension (where green, red, and blue are instances of the color dimension). The following example specifies two dimensions of the stimuli: "Indicate the color and shape of the figures appearing on the screen" (where green, red, and blue are instances of color dimension, and triangles, squares and circles are instances of the shape dimension). However, a more complex level of the rule specifies a relation among two or more dimensions, each relation forming a relational frame (Trigo, Martinez, \& Moreno, 1995). For example, in the instruction "Give me the apples that are smaller than the oranges," the speaker implies a relation between apples and oranges in terms of size dimension. This level of relationship is equivalent to the typical first order matching-to-sample procedure where behavior of the subject comes under discriminative control of a fourth-term--as in Sidman's four-term contingency (SD (SDR-SRI) (1986).

Rules of higher level of complexity, however, involve a secondary or higher-order class of relation. A second-order response then involves abstracting a relation from other relation(s). Thus, a higher order relation includes a second-order stimulus control of rules and associates one relation to other dimensions (or to other relations). This level seems to correspond to Sidman's five-te $\mathrm{rm}$ contingency ( $\mathrm{S}-\mathrm{SD}-\{\mathrm{SD}-\mathrm{R}-\mathrm{SR} \mid)$ ). There is no limit to the complexity embedded in the rule 
because it is always possible to add one more dimension or to add more relations. For instance, a third order conditional relation would include at least one second order relation, and so on.

Simple versus complex mile-governed behavior. Correspondence between the level of rules and verbally-controlled behavior is likely. Less complex rule-governed behavior more often corresponds to simpler rules; in turn, more complex behavior adjusts to higher-level contingency arrangements. For the listener to adjust or respond according to a specified rule, his or her optimal performance should ultimately correspond to the complexity of the verbal stimuli controlling his /her behavior. A concept similar to maximizing may help here. Given two or more rules provided, an individual will follow the rule with higher probability of reinforcement. In addition to the level of rule complexity, the probability that the listener will follow a rule ultimately depends on the context within which the rule is provided and the listener's history with other similar rules. Listener's history may explain the disparities in behavior among recipients of similar rules in comparable contexts. For instance, a listener may interpret an algebraic rule of moderate complexity to be simple or complex, depending upon his or her knowledge of mathematics.

\section{Sources of Rules}

Rules provided by others versus self-provided and self-generated rules. Rule identification should consider the source of the antecedent stimulus control. In cases of rules provided by others, the speaker (other than the listener) specifies, implicitly or explicitly, the criterion for the listener's behavior. In the case of self-provided rules, the speaker and the listener are the same individual. Also, self-provided rules can be taught by others or self-generated or abstracted by the subject from learning experiences. In the first case, although the rule is self-provided, it does not originate in the behavior of the subject (e.g., problem solving behavior), but in the behavior of others. The speaker/listener may have no understanding of how to arrive at, or derive such a rule, because he or she may "know that" but not "know how or why" the contingencies specified in such rules are related. Rules taught by others are often learned via imitation processes (i.e., immediate, delayed and pervasive or generalized imitation processes, see Peláez-Nogueras \& Gewirtz, 1995).
In the case of "self"-generated rules (or rule generated by the subject), a developmental history of direct experiences with at least some of the related contingencies specified in the verbal rule is required. (The term "self" as used here, does not imply the initiation of a behavior by an autonomous internal agent or by some imaginary part of the individual, it refers to the individual's behavior repertory.) The verbal contingency specifications produced by the individual allows him or her to arrive, derive, or abstract other relations. Rule generation (rule derivation or rule emergence) can occur through transfer processes of learning, as in transitivity (Sidman, 1986) and combinatorial entailment (Hayes, 1991; Hayes \& Hayes, 1992). Only after having acquired a receptive understanding of a rule and expressed an explicit rule, can the listener emit rule-corresponding behavior. When an individual can state or describe to others the orderliness of the environmental relations (the contingencies) we assume he or she "knows" the rule.

Conforming versus complying behavior. With rules provided by others, the speaker specifies the criterion for the listener's behavior, expecting the listener to adjust, conform, or behave according to rule descriptions (e.g., as in the mand). With selfprovided rules, whether previously taught by others or self-generated, the subject's ability to verbalize the rule seems to affect his or her subsequent performance on a transfer task (Peláez et al., in progress). The ability to self-state or self-provide a rule, however, may not be the sole cause of the rule-following behavior. This is due to the influence of the listener's experience with reinforcing contingencies and the nature of the specific contexts involved.

The distinction made in the literature between complying with and conforming to rules may be pertinent here (Verplanck, 1992). Rule compliance denotes following and behaving according to rules that have been either stated to the listener, or selfprovided. Rule-conforming denotes behavior consistent with the rule, although the listener may remain unable to verbalize or self-generate the rule.

\section{Taxonomy of Rules}

The taxonomy is based on four different dimensions of rules and its corresponding rule-govemed behavior. Each dimension stresses different aspects of rules and describes its potentially- rela- 
ted behavior. We examine the different dimensions of a rule in terms of accuracy, explicitness, complexity level, and source. Specifically, a rule should be described by analyzing all four dimensions involved, which will allow for a more systematic approach to the study of rule-governed behavior.

Figure 2 shows all possible types of rules (a total of 16 rules) resulting from combinations among the four different dimensions. The dimensions of a rule are presented in dichotomous fashion, even though they can operate along a continumm occurring within the four dimensions: (a) explicit vs. implicit, (b) accurate vs. inaccurate, (c) lower vs higher complexity, and (d) provided by others vs. self-provided. By deconstructing rules into their elements and examining each rule dimension individually, we attempt a more precise developmental approach to be employed in experiments where different types of rules are manipulated to determine their impact on rule-governed behavior and its progression.

The following are the 16 rules derived from a combination of the four basic dimensions discussed above:

(a) Explicit, Accurate, Lower Complexity, and Provided by Others

(b) Explicit, Inaccurate, Lower Complexity, and Provided by Others

(c) Explicit, Accurate, Higher Complexity, and Provided by Others.

(d) Explicit, Inaccurate, Higher Complexity, and Provided by Others.

(e) Explicit, Accurate, Lower Complexity, and Self-Provided

(f) Explicit, Inaccurate, Lower Complexity, and Self-Provided

(g) Explicit, Accurate, Higher Complexity, and Self-Provided

(h) Explicit, Inaccurate, Higher Complexity, and Self-Provided

(i) Implicit, Accurate, Lower Complexity, and Provided by Others

(j) Implicit, Inaccurate, Lower Complexity, and Provided by Others

(k) Implicit, Accurate, Higher Complexity, and Provided by Others

(I) Implicit, Inaccurate, Higher Complexity, and Provided by Others.

(m) Implicit, Accurate, Lower Complexity, and Self-Provided

(n) Implicit, Inaccurate, Lower Complexity, and Self-Provided

(o) Implicit, Accurate, Higher Complexity, and Self-Provided

(p) Implicit, Inaccurate, Higher Complexity, and Self-Provided.

For example, cell (a) represents an explicit, accurate rule, of lower complexity, provided by a speaker other than the listener. The parental order: "Pick up these toys now if you want to watch TV" exemplifies such a rule. This example posits a clear specification of all the components of the three-term contingency in context (is of lower complexity and lower developmental level). In this case, the verbal descriptions are provided by the parent (speaker other than the listener), and the contingencies correspond to (are congruent with) the actual contingencies encountered by the child (the listener). The last type of rule (see the right bottom cell (p) in Figure 2), represents an implicit, inaccurate, of higher complexity level and self-derived rule (the speaker and the listener being the same). A rule of this type can be found, for example, in the self-instruction, "At the party, I should approach Linda the same way that Juan approaches Mary when they are dancing--not how he approaches her when they are at school." This represents a self-provided rule, which the subject assumes to contain implicit positive consequences (i.e., acceptance). But Linda's aversion to guys renders the rule inaccurate and the real consequence will be rejection. The complexity of this rule is high because it involves a second-order conditional discrimination (i.e., it first requires approaching Linda during dancing and not approaching her during school, and second, it requires matching, that is, to behave just as Juan towards Mary). This relation requires that the subject abstracts the rule from the couple's relation (the sample stimuli) and applies it during his interaction with Linda, and only in a specific context. This type of rules represents higher complexity and developmental level.

The rule-governed behaviors derived from this taxonomy are labeled according to each type of rule governing. We are starting a program of research that focuses on investigating these taxonomy of rules from a developmental perspective, that is, in determining their hierarchical organization in learning. Our assumption is that the taxonomy of rules offered here, by ranging from explicit to implicit, lower level to higher level of complexity, accurate to inaccurate, imposed by others to self-generated, can organize behavior by increased level of difficulty, compliance, and adjustment to the contingencies they specify. 


\section{Other Related Rule Taxonomies}

Our taxonomy is not exhaustive; when employing other criteria, other taxonomies can be identified. Ply and track rules (Zettle \& Hayes, 1982) were not included in our classification because, according to our analysis, a rule should first be defined in terms of the contingencies it specifies regardless of whether the listener obeys or violates such rule. Thus, given that ply and track rules are exclusively defined in terms of their correspondence to pliance and tracking, rule-dimensions in plys and tracks cannot be identified nor manipulated (i.e., as independent variables)--independently of the specific behavior of the listener. This posits serious problems for an experimental situation, where the types of rules to be studied must be defined first. In such circular cases, an investigator would be unable to isolate and define a priori a rule and its dimensions for the purpose of experimental manipulations.

We have excluded Skinner's (1957) mand and tact from our rule taxonomy for similar reasons. Just like plys and tracks, mands and tacts are defined exclusively in terms of the listener's responses- these types of rules can not be properly identified a priori or independently of the history of the listener. Another distinction made in the literature is that between nomative and normal rules (Reese, 1989; Reese \& Fremow, 1984). In our analysis, we are only concemed with normative rules. Nonnal rules are not considered due to their lack of contingency specification and their dismissal of the listener's behavior, both necessary conditions in our taxonomy.

\section{Conclusion}

We offered a classification of 16 types of rules derived from four dimensions (i.e., explicitness, accuracy, complexity, and source) and their differential effects on listeners' behavior. Even though we assume a functional co-dependence between rules and rule-governed behavior, the taxonomy requires a separate analysis of the contingencies specified in the rule and of those related to the rule-following behavior. In studying the control that a rule exerts on rule-following behavior, one must first adequately define and identify separately the rule and the rule-following behavior. In studying behavioral development, when analyzing the various effects each rule exerts on the listener's behavior, one must consider the four dimensions of rule, the contingency history of the listener, and the context within which the rule is provided. Even though manipulations of some types of rules have been conducted in studies of self-instruction, relational frames, and derived relations, we believe that a more systematic study of the differential effects of the proposed four dimensions of rules on the listener's behavior is needed.

\section{References}

Buskist, W. F., \& Miller, H.L. (1986). Interaction between rules and contingencies in the control of human fixed-interval performance. The Psychological Record, 36 , 109-116.

Catania, A. C. (1985). Rule-governed behavior and the origins of language. In C. F. Lowe, M. Richelle, D. E. Blackman, \& C. M. Bradshaw (Eds.), Behavior analysis and contemporary psychology (pp. 135-156). London: Erlbaum.

Catania, A. C. (1998). Learning (4th ed). Upper Saddle River, NJ: Prentice Hall.

Catania, A. C., Matthews, B. A., \& Shimoff, E. (1982). Instructed versus shaped human verbal behavior: Interactions with nonverbal responding. lournal of the Experimental Analysis of Behavior, 38, 233-248.

Catania, A. C., Shimoff, E., \& Matthews, B. A (1989). An experimental analysis of rule-governed behavior. In S.C. Hayes (Ed.), Rule-governed behavior: Cognition, contingencies and instructional control (pp. 119-152) New York: Plenum Press.

Cerutti, D. T. (1989). Discrimination theory of rulegoverned behavior. lournal of the Experimental Analysis of Behavior, 51, 2 9-276.

Degrandpre, R.J., \& Buskist, W.F. (1991). Effects of accuracy of instructions on human behavior. The Psychological Record, 41, 371-384.

Galizio, M. (1979). Contingency-shaped and rulegoverned behavior: Instructional control of human loss avoidance. Journal of the Experimental Analysis of Behavior, $51,3.70$

Hayes, S. (1991). A relational control theory of stimulus equivalence. In L. J. Hayes \& P. N. Chase (Eds.), Dialogues on verbal beliavior (pp. 19-40). Reno, NV: Context Press.

Hayes, S. C. \& Hayes, L. J. (1992). Verbal relations and the evolution of behavior analysis. American Psychologist, 47, 1383-1395.

Hayes, S. C., \& Hayes, G. J. (1994). Stages of moral development as stages of rule-governance. In L. H. Hayes, G. J. Hayes, S. C. Moore, \& P. M. Ghezzi (Eds.), Ethical issues in developmental disabilities, (pp. 45-65). Reno, NV: Context Press.

Hineline, P. N., \& Wanchisen, B. A. (1989). Correlated hypothesizing and the distinction between contingency-shaped and rule-governed behavior. In S. C. Hayes (Ed.), Rule-governed behavior: Cognition, contingencies and insiructional control (pp. 221-268). New York: Plenum Press.

Kantor, J.R. (1924/1926). Principles of psychology. (Vol 1 and 2). Chicago: Principia Press.

Martínez, H., González, A., Ortiz, G., \& Carrillo, K. (in press). Aplicación de un modelo de covariación al análisis de las ejecuciones de sujetos humanos en condiciones de entrenamiento y de transferencia. Revista Latinoamericana de Psicologia.

Martínez, H., Moreno, R., Ortiz, G. \& Carrillo, K (in press). Eficiencia en la ejecución: Una aplicación del modelo de covariación. Revista de Psicologia y Ciencia Sacial.

Martinez, H., \& Ribes, E. (1996). Interactions of con- 
tingencies and instructional history on conditional discrimination. The Psychological Record, 46, 301-318.

Michel, R.L., \& Bernstein, D. J. (1991). Transient effects of acquisition history on generalization in a matching to sample task. Journal of the Experimental Analysis of Behavior, 56, 155-166.

Morris, E. K. (1988). Contextualism: The world view of behavior analysis. Journal of Experimental Child Psychology, 46, 289-323

Pelaez, M. (1994). Contextualism in behavior analysis of development: Upon further reflection. Behavioral Development, 4, 8-12.

Peláez-Nogueras, M., \& Gewirtz, J. L. (1995). The learning of moral behavior: A behavior-analytic approach. In W.M. Kurtines \& J.L. Gewirtz (Eds.), Moral behavior: An introduction (pp. 173-199). Boston: Allyn \& Bacon.

Peláez, M., Moreno, R., Martinez, R., Trigo, E., \& Qiang, A. (in review). The function of self-verbalized rules on transfer learning in a higher-order conditionaldiscrimination task.

Reese, H. W. (1989). Rules and rule-governance: Cognitive and behavioristic views. In S. C. Hayes (Ed.), Rule-governed behavior: Cognition, contingencies, and instructional control, (pp. 3-84). New York: Plenum Press.

Reese, H. W., \& Fremouw, W.J. (1984). Normal and normative ethics in behavioral sciences. American Psychologist, 39, 863-876.

Ribes, E. (1992). Some thoughts on thinking and its motivation. in S.C. Hayes \& L. J. Hayes (Eds.), Understanding verbal relations, (pp. 211-224). Reno, NV: Context Press.

Schlinger, H., \& Blakely, E. (1987). Function-altering effects of contingency-specifying stimuli. The Behavior Analyst, 10, 41-45.

Sidman, M. (1986). Functional analysis of emergent verbal classes. In T. Thompson \& M.D. Zeiler (Eds.), Analysis and integration of behavioral units (Pp 93-114). Hillsdale, NJ: Erlbaum.

Skinner, B. F. (1953). Science and human behavior. New York: Macmillan.

Skinner, B. F. (1957). Verbal behavior. Englewoods Cliffs, N\}: Prentice-Hall.

Skinner, B. F. (1966). An operant analysis of problem solving. In B. Kleinmuntz (Ed.), Problem solving: Research, method and theory. New York: Wiley.

Skinner, B. F. (1969). Contingencies of reinforcement: A theoretical analysis. New York: Appleton-Centur $y$-Crofts.

Skinner, B. F. (1989). The behavior of the listener. In S. C. Hayes (Ed.), Rule-governed behavior: Cognition, contingencies, and instructional control (pp. 85-96). NY: Plenum Press.

Trigo, E. (1998). Tareas experimentales de prueba de hipotesis: Estrategias de diseño en la tarea de selección. Dissertation manuscript. University of Seville Library, Spain.

Trigo, É., Martinez, R., \& Moreno, R. (1995). Rule performance and generalization in a matching-to-sample task. The Psychological Record, 45, 223-240.

Vaughan, M. (1989). Rule-governed behavior in behavior analysis: A theoretical and experimental history. in S. C. Hayes (Ed.), Rule-governed behavior: Cognition, contingencies, and instnictional control (pp. 97-118). New York: Plenum Press.

Verplanck, W. S. (1992). Verbal concept "mediators" as simple operants. The Analysis of Verbal Behavior, 10, 45-68.

Zettle, R. D., \& Hayes, S. C. (1982). Rule governed behavior: A potential theoretical framework for cognitive-behavior therapy. In P. C. Kendall (Ed.), Advances in cognitive-behavioral research and therapy (Vol. 1, pp. 73118). New York: Academic Press.

\section{Authors' Note:}

Portions of this article were first reported in a paper titled The Development of Rules that Control Behavior delivered by the first author at the annual meeting of the Experimental Analysis of Behavior Group, London, UK, April, 1996. The taxonomy here reported was originally published in the Mexican Journal of Behavior Analysis, 24, 1998. Appreciation is extended to Carlos Bruner, Editor of the Mexican Journal, for granting permission for publication in this Bulletin of a version of the original article. We thank Bryan Midgley, Peter Nogueras, and John Visconti for their critical reviews of an earlier version of this paper. Correspondence on this article should be addressed to Dr. Martha Peláez, Department of Educational Psychology \& Special Education, College of Education-EB 242B, Florida International University, Miami, FL, 33199, USA. (E-mail: marthapn@aol.com)

\section{Developmental SIG Dinner at ABA}

The SIG Dinner will take place on Friday, May 28, starting around 6:00p.m. and terminating before 9:00p.m. It will be held in REZA'S RESTAURANT, 432 West Ontario, Chicago. The cuisine great Persian and great. A family style dinner will be served for $\$ 22.45$ (tax \& gratuity included). The appetizers will include a vegetarian plate, hummos, and grilled mushrooms. The entrees will be filet mignon, shish kebab, seasoned ground beef, and chicken breast kebab. Tea, coffee, soft drinks and dessert are included. A cash bar for alcoholic drinks will be set up in the room. The restaurant 14-seat bus will leave at approximately 10min intervals, beginning at 5:45 p.m., from the North side of the Hilton Towers. There is no charge for the service. Please make reservations and provide advance payment to Jack Gewirtz, 2025 Brickell Ave. \# 1802, Miami, FL 33129.

We hope you can make the dinner. It was memorable two years ago. 